\title{
Квантовые поправки и магнитотранспорт в пленках 3D дираковского полуметалла $\mathrm{Cd}_{3-x} \mathrm{Mn}_{x} \mathrm{As}_{2}$
}

\author{
(ㄷ А.Б. Мехия ${ }^{1}$, А.А. Казаков ${ }^{1}$, Л.Н. Овешников ${ }^{2,1}$, А.Б. Давыдов ${ }^{1}$, А.И. Риль ${ }^{3}$, \\ С.Ф. Маренкин ${ }^{3,4}$, Б.А. Аронзон ${ }^{1}$ \\ ${ }^{1}$ Физический институт им. П.Н. Лебедева Российской академии наук, \\ 119991 Москва, Россия \\ 2 НИЦ „Курчатовский институт“, \\ 123182 Москва, Россия \\ ${ }^{3}$ Институт общей и неорганической химии им. Н.С. Курнакова Российской академии наук, \\ 119991 Москва, Россия \\ ${ }^{4}$ НИТУ „МИСиС", \\ 119049 Москва, Россия \\ E-mail: a_mehia@mail.ru
}

Поступила в Редакцию 23 мая 2019 г.

В окончательной редакции 30 мая 2019 г.

Принята к публикации 30 мая 2019 г.

Исследовались тонкие пленки твердых растворов на основе трехмерного дираковского полуметалла $\mathrm{Cd}_{3} \mathrm{As}_{2}$ с добавлением марганца. Пленки $\mathrm{Cd}_{3-x} \mathrm{Mn}_{x} \mathrm{As}_{2}(x=0,0.05$ и 0.1 ) толщиной 50-70 нм были получены на ситалловых подложках с помощью вакуумно-термического напыления из слитков арсенида кадмия, допированных Мn и изготовленных непосредственным сплавлением элементов вакуумно-ампульным методом. Были проведены измерения температурных и магнетополевых зависимостей сопротивления и определены транспортные параметры исследуемых пленок. В пленках с $x=0$ и 0.05 наблюдалось положительное магнетосопротивление характерной формы, соответствующее вкладу эффекта слабой антилокализации. При большем содержании $\mathrm{Mn}(x=0.1)$ наблюдался вклад от эффекта слабой локализации. Подобная смена типа квантовой поправки, применительно к топологическим полуметаллам, указывает на перестройку зонной структуры и переход из состояния дираковского полуметалла в фазу тривиального полупроводника, который в данном случае соответствует критическому содержанию $\mathrm{Mn} x_{c} \sim 0.07$.

Ключевые слова: ситаловые подложки, вакуумно-термическое напыление, антилокализация.

DOI: 10.21883/FTP.2019.11.48443.9168

\section{1. Введение}

Дираковские полуметаллы (ДПМ) - это системы, в которых объемные носители заряда имеют бесщелевой линейный спектр, часто называемый „конусом Дирака“ $[1,2]$. Носители заряда в такой системе представляют собой безмассовые фермионы с жесткой связью направлений импульса и спина [3]. Одним из самых известных материалов, обладающих линейным спектром, является графен [4] — двумерный кристалл, состоящий из атомов углерода, образующих гексагональную решетку. ДПМ обладают нетривиальной зонной структурой, и закон дисперсии квазичастиц в них является линейным по всем трем направлениям импульсного пространства [5], в то время как в графене (или, например, на поверхности трехмерного топологического изолятора) линейный спектр является двумерным. Поэтому довольно часто ДПМ называют трехмерными аналогами графена. Нетривиальные состояния в 3D ДПМ являются топологически защищенными, что гарантирует их устойчивость по отношению к немагнитным примесям и дефектам.

Нарушение $T$ - или $P$-симметрии (симметрии по отношению к обращению времени и инверсии координат соответственно) в ДПМ приводит к снятию исходного вырождения дираковских точек и переводит систему в состояние вейлевского полуметалла [5]. При этом спин-поляризованные поверхностные состояния в такой системе образуют фермиевские арки в импульсном пространстве $[5,6]$, попарно соединяющие проекции объемных вейлевских точек противоположной киральности. Свойства вейлевского полуметалла зависят от того, какой именно тип симметрии нарушен. В частности, в системах с нарушенной $T$-симметрией должен наблюдаться аномальный эффект Холла, особенно выраженный в случае, когда химический потенциал находится вблизи вейлевских точек [1]. Таким образом, ДПМ системы являются уникальной платформой для исследования переходов между различными топологическими фазами, индуцируемых различными воздействиями, как например изменением химического состава материала или приложением одноосных деформаций.

Не так давно фаза трехмерного ДПМ была обнаружена в соединениях $\mathrm{Na}_{3} \mathrm{Bi}$ и $\mathrm{Cd}_{3} \mathrm{As}_{2}$ [3]. Методом фотоэлектронной спектроскопии с угловым разрешением было показано, что $\mathrm{Cd}_{3} \mathrm{As}_{2}$ обладает всеми перечисленными выше особенностями электронной структуры [7], что также подтверждается результатами зон- 
ных расчетов из первых принципов [8]. Кроме того, в $\mathrm{Cd}_{3} \mathrm{As}_{2}$ наблюдался целый ряд необычных явлений, включая киральную аномалию, гигантское магнетосопротивление, а также недавно обнаруженную нетривиальную сверхпроводимость $[9,10]$. В отличие от $\mathrm{Na}_{3} \mathrm{Bi}$, кристаллы $\mathrm{Cd}_{3} \mathrm{As}_{2}$ стабильны на воздухе, что делает их более привлекательными для исследования. Помимо этого, $\mathrm{Cd}_{3} \mathrm{As}_{2}$ привлекает значительное внимание, так как обладает высокой подвижностью носителей заряда даже при комнатной температуре [11]. Стоит отметить, что роль поверхностных состояний растет при уменьшении одного из характерных размеров системы (толщины), приводя к появлению у системы новых свойств. В недавней работе было показано, что для пленок $\mathrm{Cd}_{3} \mathrm{As}_{2}$ толщиной менее 70 нм, выращенных на подложках $\mathrm{CdTe}$ методом молекулярно-лучевой эпитаксии, уже при $\sim 10$ Тл наблюдается квантовый эффект Холла [12]. Это дает нам надежду обнаружить аналогичные эффекты при дальнейших исследованиях в сильных полях.

\section{2. Синтез и идентификация образцов}

Для измерения были выбраны образцы $\mathrm{Cd}_{3-x} \mathrm{Mn}_{x} \mathrm{As}_{2}$ с содержанием марганца $x=0,0.05$ и 0.1 . Пленки толщиной $\sim 50-70$ нм были синтезированы методом вакуумно-термического напыления из объемных кристаллов соответствующих составов, размерами 1-2 мм. Использованные кристаллы были выращены из высокочистых элементов $\mathrm{Cd}, \mathrm{Mn}$ и $\mathrm{As}$, непосредственным сплавлением вакуумно-ампульным методом. Синтез проводили в 3 этапа с учетом того, что в состав арсенида кадмия входит легко летучий элемент As. Первый этап состоял в нагреве до $620^{\circ} \mathrm{C}$ со скоростью $30^{\circ} \mathrm{C} /$ ч и дальнейшей выдержке при этой температуре в течение 5 ч. На втором этапе производили нагрев до $780^{\circ} \mathrm{C}$ со скоростью $20^{\circ} \mathrm{C} /$ ч и выдержкой в течение 2 сут для гомогенизации расплава. На третьем этапе проводили охлаждение ампулы в режиме выключенной печи.

Синтез кристаллов проводили в электропечах с использованием температурных регуляторов ТЕРМОДАТ (точность поддержания температуры составляла $\pm 1^{\circ} \mathrm{C}$ ) с компьютерным управлением и режимом записи данных, в двойных вакуумированных (до уровня $\sim 10^{-2}$ Па) кварцевых ампулах. Для уменьшения эффекта переноса в свободный объем ампулы легколетучего As ампула была помещена в изотермическую зону печи. Внутренняя поверхность ампулы подвергалась графитизации, чтобы исключить взаимодействие стенок ампулы с расплавами. Температурно-временнб́е режимы синтеза подбирались с учетом высоких давлений паров мышьяка. Для хорошей гомогенизации проводилась длительная выдержка расплавов.

Вакуумно-термическое напыление исследуемых образцов проводилось в установке ВУП-5. Давление остаточных паров составляло не более $1 \cdot 10^{4}$ Па. В качестве

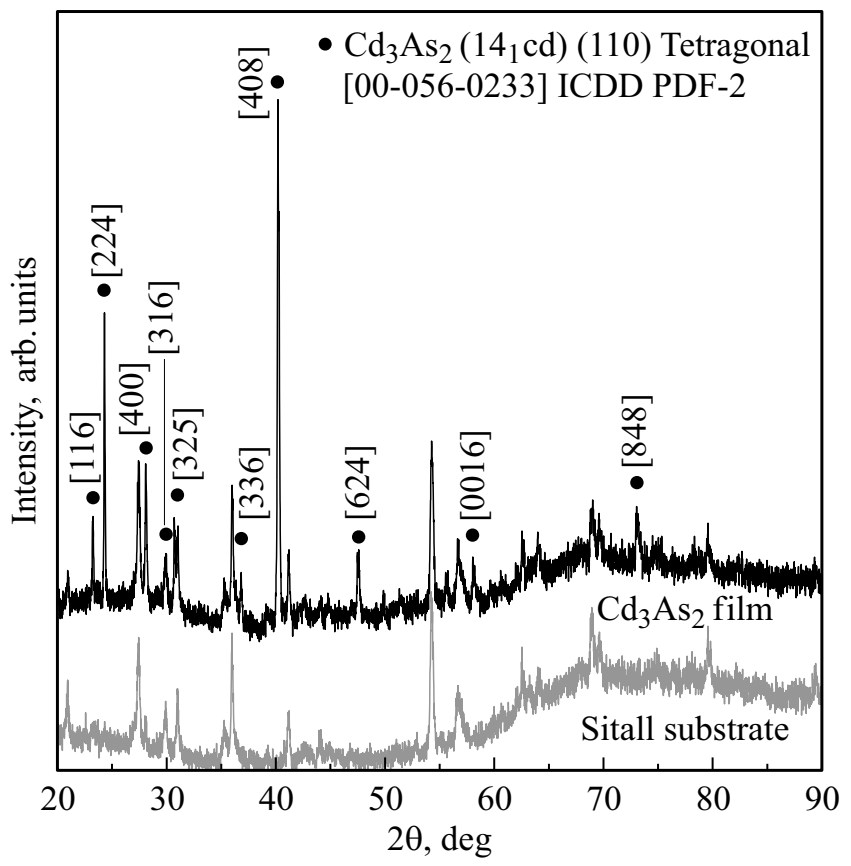

Рис. 1. Результаты РФА для исследуемых пленок $\mathrm{Cd}_{3} \mathrm{As}_{2}$.

подложек использовались ситалловые пластины марки CT-50-1, размером $3 \times 5$ мм. Испарение мишени (объемных кристаллов $\left.\mathrm{Cd}_{3-x} \mathrm{Mn}_{x} \mathrm{As}_{2}\right)$ проводилось из вольфрамового испарителя резистивного типа. Расстояние между испарителем и подложками составляло 4 см. Температура подложек при синтезе составляла $300 \mathrm{~K}$. Расположение испарителя и подложек относительно друг друга было выбрано осесимметричным. Процесс синтеза пленок проводился до полного испарения материала мишени.

Идентификацию полученных пленок проводили с помощью рентгенофазового анализа (РФА) (рис. 1) в Центре коллективного пользования ИОНХ РАН на дифрактометре BRUKER D8 ADVANCE (излучение $\mathrm{Cu} K_{\alpha}$, $\lambda=0.1540 \mathrm{нм}, U=40 \mathrm{\kappa B}, I=40 \mathrm{MA})$. Съемку производили с шагом $0.014^{\circ}$ в диапазоне $2 \Theta: 10-90^{\circ}$ и выдержкой в каждой точке $1.3 \mathrm{c}$. При обработке полученных рентгенограмм использовалась база порошковых дифрактометрических данных ICDD PDF-2 и программные среды Diffrac.SuiteEVA и Topas.

Как видно из представленной дифрактограммы (рис. 1), в составе исследуемой пленки присутствует только фаза $\alpha-\mathrm{Cd}_{3} \mathrm{As}_{2}$ тетрагональной объемно центрированной модификации $\mathrm{I} 4_{1} \mathrm{~cd}$, часть пиков которой совпадает с пиками самой подложки ситалла, но при этом никаких следов сторонних (примесных) фаз на дифрактограмме не наблюдается. Небольшая относительная интенсивность регистрируемых пиков и достаточно малое соотношение сигнал/шум не позволили провести комплексный анализ полученных дифрактограмм, что является частым ограничением при исследовании большинства пленочных образцов. 


\section{3. Результаты магнитотранспортных измерений}

Для исследуемых пленок $\mathrm{Cd}_{3-x} \mathrm{Mn}_{x} \mathrm{As}_{2}(x=0,0.05$ и 0.1) были получены температурные и магнетополевые зависимости компонент тензора сопротивлений. Образец охлаждался от комнатной температуры до температуры жидкого гелия $(4.2 \mathrm{~K})$, скорость охлаждения поддерживалась с помощью термоконтроллера на уровне $10 \mathrm{~K} /$ мин. Полученные температурные зависимости сопротивления исследуемых образов приведены на рис. 2, $a$.

Во всем исследованном диапазоне температур характер зависимости остается полупроводниковым для всех образцов серии. На рис. $2, b$ можно видеть, что полученная зависимость, перестроенная в координатах $\ln (\rho)(1 / T)$, становится близка к линейной. Это можно интерпретировать как переход к активационному режиму проводимости при $T \leq 25 \mathrm{~K}$.
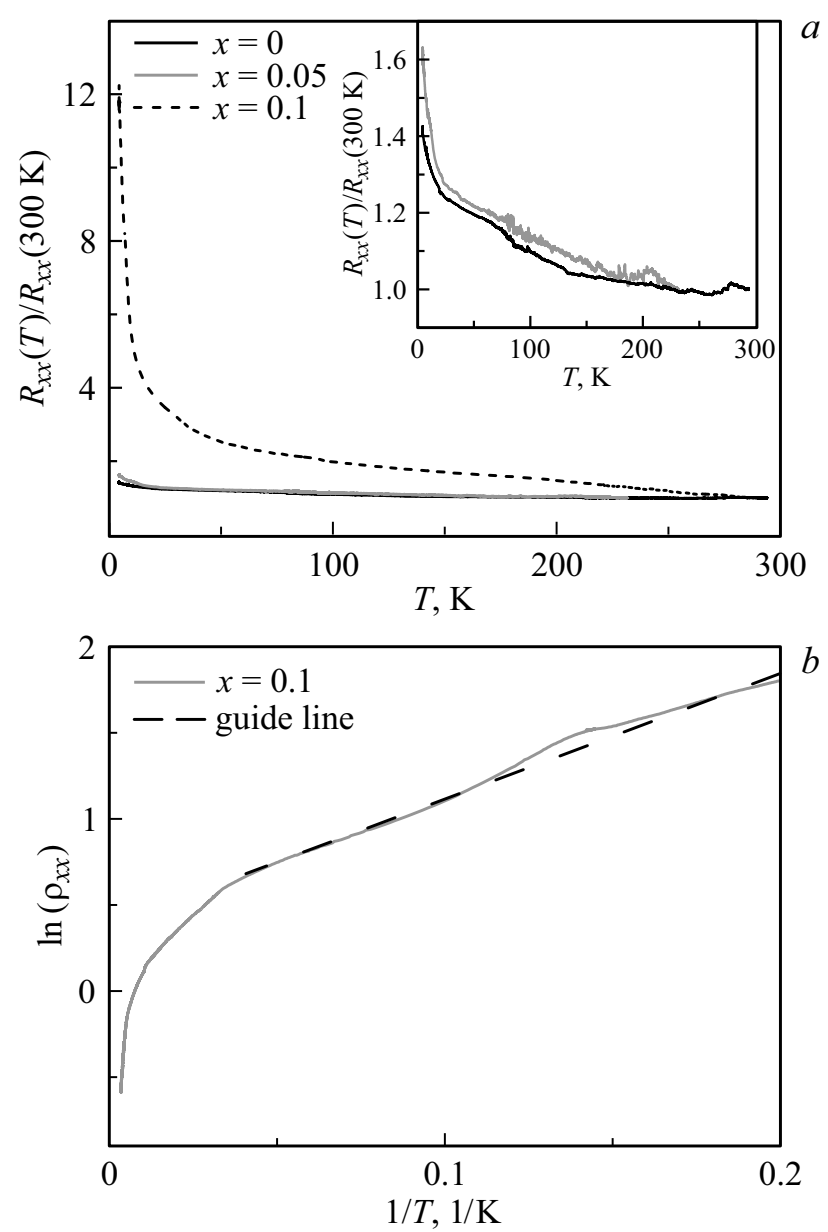

Рис. 2. $a-$ температурные зависимости сопротивления образцов $\mathrm{Cd}_{3-x} \mathrm{Mn}_{x} \mathrm{As}_{2} \mathrm{c} x=0.1$ (сплошная линия), $x=0.05$ (точки) и $x=0$ (штрихи). На вставке - температурная зависимость сопротивления образцов с $x=0.05$ и $x=0$ в увеличенном масштабе. $b$ - температурная зависимость сопротивления в координатах $\ln (\rho)(1 / T)$. Ниже $T \sim 30 \mathrm{~K}$ зависимость становится близка к линейной.
Таблица 1. Холловские параметры исследуемой серии образцов при $T=4.5 \mathrm{~K}$

\begin{tabular}{l|c|c|c|c}
\hline Образец & $R_{\mathrm{H}}$, Ом/Тл & $n, \mathrm{~cm}^{-3}$ & $\rho_{0}$, Ом $\cdot \mathrm{cm}$ & $\mu, \mathrm{cm}^{2} /(\mathrm{B} \cdot \mathrm{c})$ \\
\hline$x=0$ & 41.2 & $2.87 \cdot 10^{18}$ & 0.040 & 102 \\
$x=0.05$ & 79.9 & $1.15 \cdot 10^{18}$ & 0.64 & 12 \\
$x=0.1$ & 30 & $2.1 \cdot 10^{18}$ & 6.69 & 0.45
\end{tabular}

Измерение магнитополевых зависимостей продольного и холловского сопротивлений для исследуемой серии образцов проводилось в диапазоне слабых магнитных полей (до 2 Тл) в поперечной геометрии (магнитное поле перпендикулярно плоскости образца). Зависимости холловского сопротивления оказались линейны по магнитному полю для всех образцов. Из полученных значений холловских констант были определены значения концентраций и подвижностей носителей заряда в исследуемых пленках. Полученные результаты приведены в табл. 1.

Из представленных результатов можно видеть, что увеличение содержания $\mathrm{Mn}$ приводит к сильному падению подвижности носителей заряда, при этом концентрации носителей для всех образцов оказываются относительно близки. Концентрация носителей с температурой меняется незначительно.

На рис. 3 представлены кривые магнетосопротивления (МC) для исследуемых образцов, полученные при различных температурах. Как видно из рис. $3, a$ и $b$, для образцов с $x=0$ и 0.05 в МС проявляется вклад характерной формы, связанный с эффектом слабой антилокализации (САЛ) (положительное МС). Для образца с $x=0.1$ (рис. 3, $c$ ) вместо положительного наблюдается отрицательное МC, указывающее на вклад эффекта слабой локализации $(\mathrm{CЛ})$.

В общем случае эффект СЛ возникает в результате интерференции электронной волны в замкнутом контуре, образованном упругими рассеивателями. В отсутствие неупругого рассеяния электронная волна сохраняет начальную фазу, что приводит к конструктивной интерференции волн, обходящих контур в разных направлениях. Однако, за счет наличия дираковской точки в спектре ДПМ, такой обход контура также сопровождается набегом геометрической фазы, также называемой фазой Берри. Для топологически нетривиальных систем фаза Берри равна $\pi$, что делает описанную интерференцию деструктивной и приводит к эффекту САЛ (в то время как эффект СЛ полностью подавляется). На этом основании можно утверждать, что в образце с $x=0.1$ (рис. 3, c) фаза дираковского полуметалла оказывается разрушенной. Исходя из этого можно предполагать, что увеличение концентрации $\mathrm{Mn}$ (от 0.05 до 0.1) индуцирует перестройку зонной структуры и переход от фазы ДПМ в состояние тривиального полупроводника. В рамках такого рассмотрения, критическое содержание 

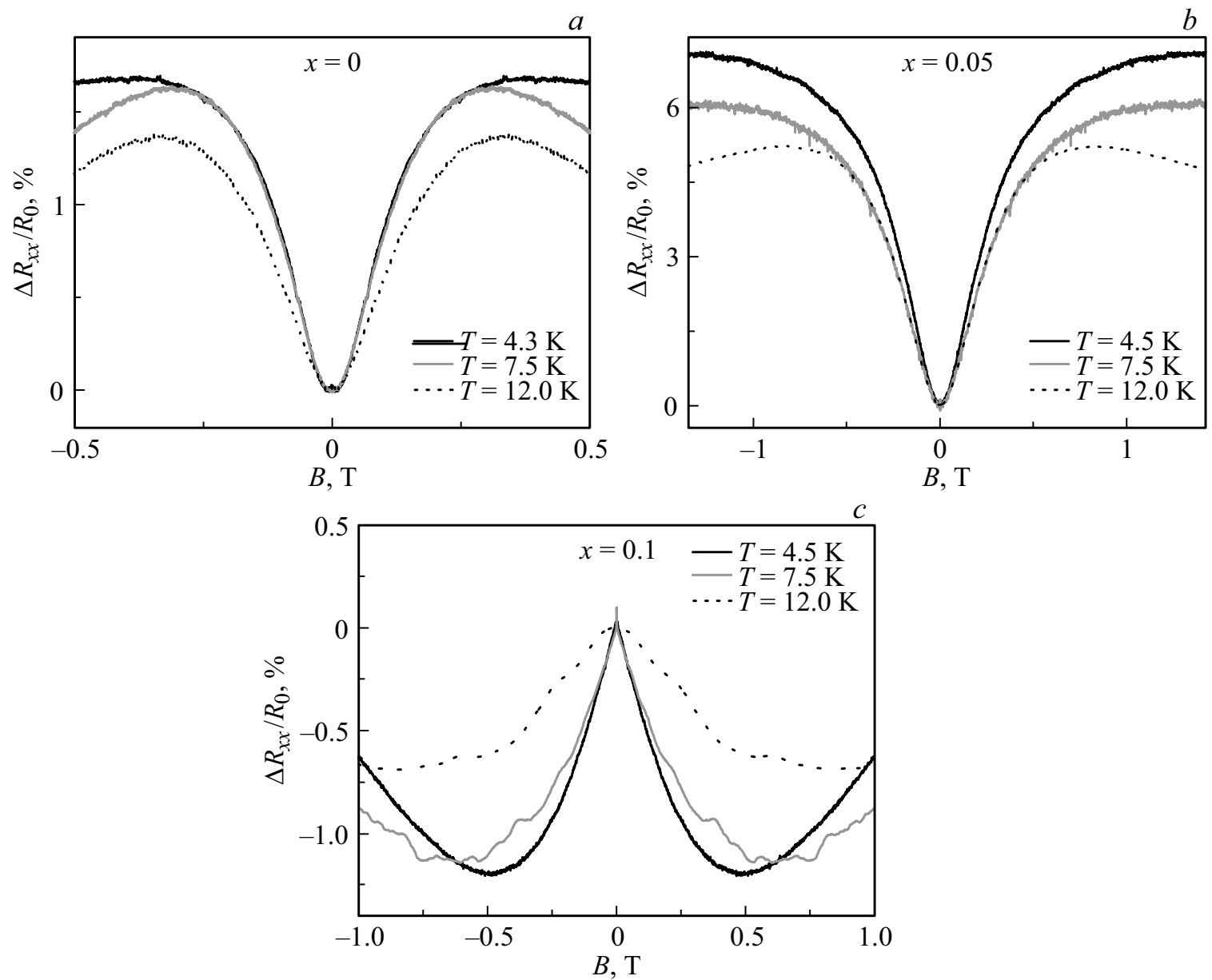

Рис. 3. Поперечное магнетосопротивление пленок $\mathrm{Cd}_{3-x} \mathrm{Mn}_{x} \mathrm{As}_{2}$ с содержанием $\mathrm{Mn}: a-x=0, b-x=0.05, c-x=0.1$. Для удобства на графиках приведено относительное изменение сопротивления $\Delta R_{x x} / R_{0}\left(R_{0}-\right.$ сопротивление образца в нулевом поле).

$\mathrm{Mn}$, соответствующее переходу, можно оценить как $x_{c} \sim 0.07$.

Учитывая низкие значения подвижностей носителей заряда, размерность исследуемых систем в рамках теории квантовых поправок является неочевидной, поэтому мы обрабатывали кривые и по формулам для двумерного и для трехмерного случаев.

Полученные кривые МС, пересчитанные в магнетопроводимость, аппроксимировались по стандартной формуле, описывающей квантовые поправки в трехмерных системах [13]:

$$
\Delta\left(\frac{1}{\rho_{x x}}\right)_{3 D}=\alpha \frac{e^{2}}{2 \pi^{2} \hbar} \frac{\overline{e B}}{\hbar} f\left(\frac{\hbar}{4 e l_{\varphi}^{2} B}\right),
$$

где

$f(x)=\sum_{n=0}^{\infty}\left(2[\sqrt{n+1+x}-\sqrt{n+x}]-(n+x+1 / 2)^{-1 / 2}\right)$,

$\hbar$ - постоянная Планка, $e-$ заряд электрона, $B-$ внешнее магнитное поле, $l_{\varphi}$ - длина дефазировки, $\alpha$ - префактор, значение которого зависит от типа поправки. Для удобства расчета функционального ряда использовалась приближенная формула [14]:

$$
\begin{aligned}
f(x) \approx 2 & {[\sqrt{2+x}-\sqrt{x}]-\left|\left(\frac{1}{2}+x\right)^{-1 / 2}+\left(\frac{3}{2}+x\right)^{-1 / 2}\right| } \\
& +\frac{1}{48}(2.03+x)^{-3 / 2}
\end{aligned}
$$

которая в данном случае имела очень малое расхождение с формулой для полного ряда $\sim 0.01 \%$. Примеры аппроксимации кривых магнетопроводимости представлены на рис. 4.

Аппроксимация экспериментальных кривых формулой Хиками-Ларкина-Нагаоки для двумерного случая [15]:

$\Delta\left(\frac{1}{\rho_{x x}}\right)_{2 D}=\alpha \frac{e^{2}}{2 \pi^{2} \hbar}\left(\psi\left(\frac{1}{2}+\frac{1}{B} \frac{\hbar}{4 e l_{\varphi}^{2}}\right)-\ln \left(\frac{1}{B} \frac{\hbar}{4 e l_{\varphi}^{2}}\right)\right)$,

где $\psi(x)$ - дигамма-функция. Полученные параметры подгонок просуммированы в табл. 2.

В обоих случаях аппроксимация проводилась со свободным значением префактора. Результаты подгонки 

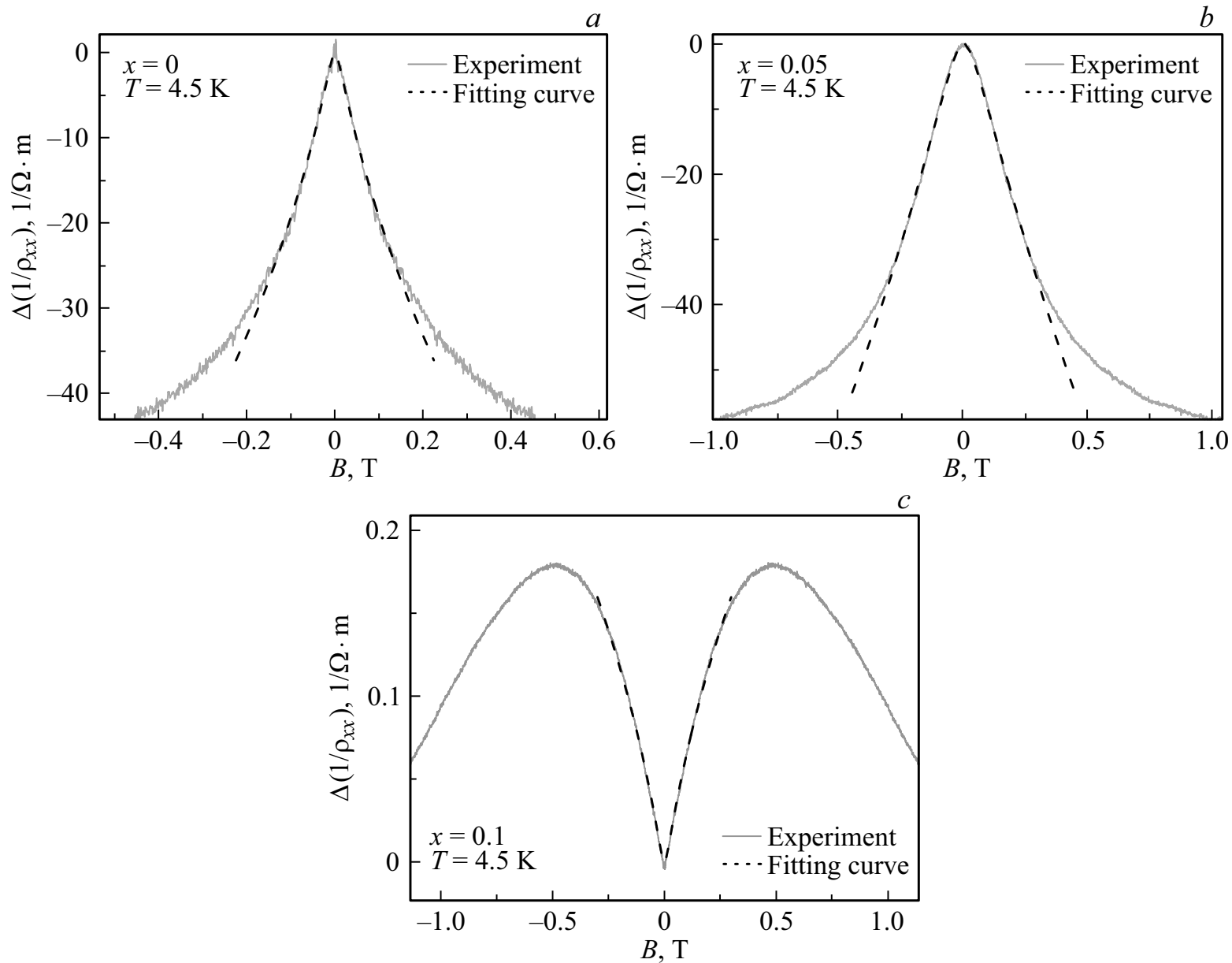

Рис. 4. Аппроксимация кривых магнетопроводимости по формуле (1) для образцов $x=0(a), x=0.05(b), x=0.1(c)$. Подгоночная кривая показана пунктиром.

Таблица 2. Параметры аппроксимации экспериментальных кривых магнетопроводимости по формулам (1) (для 3D систем) и (3) (для 2D систем)

\begin{tabular}{l|c|c|c|c}
\hline Образец & $T, \mathrm{~K}$ & $\begin{array}{c}\text { Длина } \\
\text { дефазировки } \\
\text { (3D) } l_{\varphi}, \mathrm{HM}\end{array}$ & $\begin{array}{c}\text { Префактор, } \\
\alpha\end{array}$ & $\begin{array}{c}\text { Длина } \\
\text { дефазировки } \\
(2 \mathrm{D}) l_{\varphi}, \mathrm{HM}\end{array}$ \\
\hline \multirow{3}{*}{$x=0$} & 4.5 & 146.3 & -0.4 & 108.6 \\
& 7.5 & 145.6 & -0.01 & 107.7 \\
& 12 & 127.4 & -0.02 & 77.5 \\
& 4.5 & 90.6 & -0.58 & 65.6 \\
$x=0.05$ & 7.5 & 82.6 & -0.56 & 61.8 \\
& 12 & 78.7 & -0.2 & 59.3 \\
& 4.5 & 122.2 & 0.92 & 104.7 \\
& 7.5 & 138.8 & 0.86 & 114.4 \\
& 12 & 125.9 & 0.74 & 101.0
\end{tabular}

с фиксированным значением префактора давали существенное расхождение с экспериментом для образца с $x=0$, в остальных случаях расхождение в параметрах подгонки при использовании переменного и фикси- рованного префакторов составило не более $20 \%$. Из данной таблицы можно видеть, что значение длины дефазировки несколько падает с ростом температуры или увеличением содержания Мn. Параметры, полученные при аппроксимации по формулам (1) и (3), различаются на $\sim 50 \%$ для образцов с $x=0$ и 0.05 , а для образца с $x=0.1$ - на $\sim 20 \%$. Важно отметить, что все полученные значения $l_{\varphi}$ оказываются больше или близки к средней толщине исследуемых пленок, что соответствует 2D пределу в теории квантовых поправок. Изменение знака префактора для образца с $x=0.1$ иллюстрирует смену типа поправки и указывает на переход в тривиальную фазу исследуемых пленок. Из изложенного можно заключить, что данный переход происходит при критическом содержании $\mathrm{Mn} x_{c} \sim 0.07$.

\section{4. Заключение}

Были синтезированы пленки твердых растворов $\mathrm{Cd}_{3-x} \mathrm{Mn}_{x} \mathrm{As}_{2}$ и проведена их структурная характеризация, показавшая, что кристаллическая структура пленок соответствует фазе $\alpha-\mathrm{Cd}_{3} \mathrm{As}_{2}$. Охлаждение образцов от 
комнатной до гелиевой температуры сопровождалось монотонным ростом их сопротивления. Анализ низкотемпературного магнетосопротивления выявил вклады, соответствующие квантовым поправкам к проводимости, для образцов с $x=0$ и 0.05 , соответствующих антилокализации. Увеличение содержания марганца приводит к смене знака поправки, и для образца с $x=0.1$ наблюдается слабая локализация. Согласно стандартным теоретическим представлениям, такая смена знака может указывать на переход электронной подсистемы из фазы дираковского полуметалла в состояние тривиального полупроводника при критическом содержании $\mathrm{Mn}$ $x_{c} \sim 0.07$.

\section{Финансирование работы}

Исследование транспортных свойств выполнено при поддержке РНФ (грант № 17-12-01345). Образцы получены С.Ф. Маренкиным. и А.И. Риль в рамках государственного задания ИОНХ РАН в области фундаментальных научных исследований при частичной поддержке программы Президиума РАН I.35 „Научные основы создания новых функциональных материалов““.

\section{Конфликт интересов}

Авторы заявляют, что у них нет конфликта интересов.

\section{Список литературы}

[1] N.P. Armitage, E.J. Mele, A. Vishwanath. Rev. Mod. Phys., 90 (1), 015001 (2018).

[2] S. Borisenko, Q. Gibson, D. Evtushinsky, V. Zabolotnyy, B. Buechner, R.J. Cava. Phys. Rev. Lett., $113(2), 027603$ (2014).

[3] Z.K. Liu, J. Jiang, B. Zhou, Z.J. Wang, Y. Zhang. Nature Materials, 13, 677 (2014).

[4] A.H. Castro Neto, F. Guinea, N.M.R. Peres, K.S. Novoselov, A.K. Geim. Rev. Mod. Phys., 81 (1), 1209 (2009).

[5] Zh. Wang, H. Weng, Q. Wu, X. Dai, Zh. Fang. Phys. Rev. B, $\mathbf{8 8}(12), 125427$ (2013).

[6] Siangan Wang, Ari M. Turner, A. Viswanath, S.Y. Savrasov. Phys. Rev. B, 83 (20), 205101 (2011).

[7] M. Neupane, Sui-Yang Xu, R. Sankar, N. Alidoust, G. Bian, Ch. Liu, I. Belopolski, T.-T. Cjang, H.-T. Jeng, H. Lin, A. Bansik, F. Chou, M.Z. Hasan. Nature Commun., 5, 3786 (2014).

[8] Z. Wang, H. Weng, Q. Wu, X. Dai, Z. Fang. Phys. Rev. B, 88, 125427 (2013).

[9] O.O. Shvetsov, V.D. Esin, A.V. Timonina, N.N. Kolesnikov, E.V. Deviatov. Phys. Rev. B, 99, 125305 (2019).

[10] A.V. Suslov, A.B. Davydov, L.N. Oveshnikov, L.A. Morgun, K.I. Kugel, V.S. Zakhvalinskii, E.A. Pilyuk, A.V. Kochura, A.P. Kuzmenko, V.M. Pudalov, B.A. Aronzon. Phys. Rev. B, 99, 094512 (2019).

[11] E. Zhang, Y. Liu, W. Wang, C. Zhang, P. Zhou, Z.-G. Chen, J. Zou, F. Xiu. ACS Nano, 9, 8843 (2015).
[12] M. Goyal, L. Galletti, S. Salmani-Rezaie, T. Schumann, D.A. Kealhofer, S. Stemme. APL Materials, 6 (2), 026105 (2018).

[13] B.L. Altshuler, A.G. Aronov, A.I. Larkin, D.E. Khmel'nitzkii. JETP, 54 (2), 411 (1981).

[14] D.V. Baxter, R. Richter, M.L. Trudeau, R.W. Cochrane, J.O. Strom-Olsen. J. Phys. (France), 50, 1673 (1989).

[15] S. Hikami, A.I. Larkin, Y. Nagaoka. Prog. Theor. Phys., 63, 707 (1980).

Редактор Г.А. Оганесян

\section{Quantum corrections and magnetotransport in 3D dirac semimetal $\mathrm{Cd}_{3-x} \mathbf{M n}_{x} \mathrm{As}_{\mathbf{2}}$ films}

\author{
A.B. Mekhiya' ${ }^{1}$, A.A. Kazakov' ${ }^{1}$, L.N. Oveshnikov' ${ }^{2,1}$, \\ A.B. Davydov' ${ }^{1}$, A.I. Ril' ${ }^{3}$, S.F. Marenkin ${ }^{3,4}$, \\ B.A. Aronzon ${ }^{1}$ \\ ${ }^{1}$ Lebedev Physical Institute, \\ Russian Academy of Sciences, \\ 119991 Moscow, Russia \\ ${ }^{2}$ NRC „Kurchatov institute“, \\ 123182 Moscow, Russia \\ ${ }^{3}$ Kurnakov Institute of General \\ and Inorganic Chemistry, \\ Russian Academy of Sciences, \\ 119991 Moscow, Russia \\ ${ }^{4}$ NUST MISIS, 119049 Moscow, Russia
}

Abstract In this work we investigated thin films of solid solutions based on the three-dimensional Dirac semimetal $\mathrm{Cd}_{3} \mathrm{As}_{2}$ with $\mathrm{Mn}$. The $\mathrm{Cd}_{3-x} \mathrm{Mn}_{x} \mathrm{As}_{2} \quad(x=0,0.05$ and 0.1$)$ films with thickness around $50-70 \mathrm{~nm}$ were synthesized on sitall substrates via vacuum thermal evaporation of Mn-doped cadmium arsenide ingots, obtained by the direct melting of components in vacuum ampoules. We measured temperature and magnetic field dependences of films resistivity and defined corresponding transport parameters. Films with $x=0$ and 0.05 exhibited positive magnetoresistance with characteristic form, corresponding to the contribution of weak antilocalization effect. At higher Mn content $(x=0.1)$ we observed the contribution of weak localization effect. Such change of the quantum correction type, in case of topological semimetals, suggest the transformation of the band structure and transition from the Dirac semimetal state into trivial phase, which, in our case, corresponds to the critical Mn content of about $x_{c} \sim 0.07$. 